\title{
REGULAR $\mathscr{D}$-CLASSES IN MEASURE SEMIGROUPS(1) \\ BY
}

H. S. COLLINS AND R. J. KOCH

1. Introduction. In this paper we investigate further a class of compact semigroups $S$ in which each compact simple subsemigroup is a group. We will be especially concerned with properties relating such a semigroup with its convolution semigroup of measures. It has already been shown in the paper numbered [3] that the requirement that each compact simple subsemigroup of $S$ be a group is equivalent to each of (a) the mapping $\mu \rightarrow$ carrier $\mu$ is one-one on $E(\tilde{S})$, the set of idempotents of the measure semigroup $\tilde{S}$, (b) the carrier of each idempotent in $\tilde{S}$ is a group, and (c) the compact groups of $\tilde{S}$ have the Glicksberg structure (condition (5) of Theorem 1 below). Among the further equivalences introduced here (in \$3) are (d) each regular element of $S$ has a unique inverse, and (e) there is a mapping + on $E \cdot E$ to $S$ (where $E$ is the set of idempotents of $S$ ) satisfying (i) $(e f)^{+} \in f S e$ for each $e, f \in E$, and (ii) $e^{+}=e$ for each $e \in E$. We call such a mapping a quasi-involution on $S$. If $S$ admits a quasi-involution we say that $S$ is a quasi-involution semigroup, or that $S$ has q.i. We show (Theorem 4) that $S$ has q.i. if and only if $\tilde{S}$ has q.i. The remainder of $\S 3$ is concerned with some relations between the $\mathscr{D}$-class structures of $S$ and of $\tilde{S}$. For example (Theorem 5) we show that $S$ has q.i. if and only if the carrier mapping is 1-1 modulo $\mathscr{H}$-classes. $\$ 4$ is devoted to a discussion of some stronger types of involutions motivated by the natural involution of an inverse semigroup.

2. Definitions. Throughout the paper $S$ will be a compact Hausdorff topological semigroup. The convolution semigroup $\tilde{S}$ of non-negative, regular, normalized, Borel measures on $S$ is known to be a compact Hausdorff topological semigroup if given the weak-* topology (a net $\mu_{\alpha}$ in $\tilde{S}$ converges to $\mu \in \tilde{S}$ iff $\mu_{\alpha}(f)$ $\rightarrow \mu(f)$ for each continuous complex $f$ on $S)$. Further if one defines carrier $\mu$ (for $\mu \in \tilde{S}$ ) as the least closed set in $S$ with $\mu$ measure 1, then carrier $(\mu v)=$ (carier $\mu$ ) $($ carrier $v$ ) for each $\mu, v \in \tilde{S}$. For these and related results concerning $\tilde{S}$, see [6]. A fact of fundamental importance for us is the following theorem proved in [4]: The carrier $H$ of an idempotent $\mu \in \tilde{S}$ is a compact simple semigroup (i.e., $H$ has no proper ideals) and for each continuous complex $f$ on $S$, the mapping $y \rightarrow \int f(x y) d \mu(x)$ is constant on each minimal left ideal of $H$.

An element $a$ of $S$ is regular if $a \in a S a$. An inverse of $a$ is an element $b$ of $S$

Received by the editors August 25, 1961.

(1) This work was done under NSF Grants G17777 and G14085. 
satisfying $a b a=a$ and $b a b=b$. It is known (and easy to verify) that $a$ is regular if and only if $a$ has an inverse; in fact if $a=a x a$, then xax is an inverse of $a$. Further if $b$ is an inverse of $a$, then both $a b$ and $b a$ are idempotents. Green [7] defined in $S$ the equivalence relations $\mathscr{L}$ and $\mathscr{R}$ defined as follows: $a \mathscr{L} b$ iff $a \cup S a$ $=b \cup S b ; a \mathscr{R} b$ iff $a \cup a S=b \cup b S$. He showed that $\mathscr{L}$ and $\mathscr{R}$ are permutable and hence that their relative product $\mathscr{D}$ is an equivalence relation: $a \mathscr{D} b$ iff either (and these are equivalent) (1) there exists $c \in S$ such that $a \mathscr{L} c$ and $c \mathscr{R} b$ or (2) there exists $d \in S$ such that $a \mathscr{R} d$ and $d \mathscr{L} b$. The intersection of $\mathscr{L}$ and $\mathscr{R}$ is denoted by $\mathscr{H}: a \mathscr{H} b$ iff $a \mathscr{R} b$ and $a \mathscr{L} b$. For $a \in S$ we denote by $R_{a}, L_{a}, D_{a}$, and $H_{a}$ the respective $\mathscr{R}-, \mathscr{L}-, \mathscr{D}-$, and $\mathscr{H}$ - classes to which $a$ belongs. If $a$ is regular then each element of $D_{a}$ is regular. For further information along these lines the reader is referred to [9]; for general background information on topological semigroups see [10]. We denote by $E$ the set of idempotents of $S$, and by $E(\tilde{S})$ the set of idempotents of $\tilde{S}$. We abbreviate carrier $\mu$ by $C(\mu)$.

3. Quasi-involution semigroups. In his proof of the existence of Haar measure on a compact group, Wendel [11] made use of the existence of the involution $\mu^{*}(f)=\int f\left(x^{-1}\right) d \mu(x)$ on $\tilde{S}$. He used also the fact that the partial order $\geqq$ on $\widetilde{S}$ defined by $\mu \geqq v$ iff $\mu \nu=v \mu=\mu$ is directed when restricted to $E(\widetilde{S})$. Rephrasings of these facts will be found here. Because of the large number of equivalent conditions encountered we find it expedient to include them in several theorems. We state first without proof a portion of the main result of [3]. It is emphasized that $S$ will be henceforth a compact semigroup.

THEOREM 1. The following are equivalent for a compact semigroup $S$ :

(1) $C(\mu)$ is a group for each idempotent $\mu$ in $\tilde{S}$.

(2) No three idempotents of $\tilde{S}$ are collinear.

(3) Each compact simple semigroup of $S$ is a group.

(4) The mapping $\mu \rightarrow C(\mu)$ is one-one on $E(\tilde{S})$.

(5) Each compact group in $\tilde{S}$ consists of the G-translates of Haar measure on a compact normal subgroup of some compact group $G$ of $S$.

THEOREM 2. The following are equivalent:

(1) For any idempotents $e$ and $f$ in $S$ there is an idempotent $k$ such that $\left\{(e f)^{n}\right\}$ and $\left\{(f e)^{n}\right\}$ converge to $k$.

(2) Each regular element of $S$ has a unique inverse.

(3) Each compact simple semigroup of $S$ is a group.

(4) The closed semigroup $S(e, f)$ generated by idempotents $e$ and $f$ of $S$ has a zero.

Proof. (1) implies (2). Let $a$ be a regular element. By [9, Corollary 2] a necessary and sufficient condition for $a$ to have a unique inverse is that $L_{a} \cap E$ and $R_{a} \cap E$ each reduce to a single element. Let $e, f \in L_{a} \cap E$; then $S a=S e=S f$, 
so $e f=e$ and $f e=f$. It follows, using (1), that $e=\lim (e f)^{n}=k=\lim (f e)^{n}=f$, so $L_{a} \cap E$ is a point. Similarly $R_{a} \cap E$ is a point and (2) holds.

(2) implies (3). Let $H$ be a compact simple semigroup in $S$, and let $L$ be a minimal left ideal of $H$. If $e, f \in L \cap E$, then $H e=H f$, so $e=e f$ and $f=f e$. It follows that $f$ is an inverse for $e$, so by (2) we conclude that $e=f$. Hence $L$ has a unique idempotent, and it follows [2] that there is a unique minimal right ideal $R$. Similarly there is a unique minimal left ideal $L$, and $H=L \cap R$ is a group [2].

(3) implies (4). Let $\Gamma(x)=\left\{x^{n}: n \geqq 1\right\}^{*}$. It is known that $\Gamma(x)$ has a minimal ideal $K(x)$ which is a group, that $K(x)$ is the set of cluster points of $\left\{x^{n}\right\}$, and $\Gamma(x)$ has a unique idempotent [8]. It is easy to verify that $S(e, f)=\Gamma(e) \cup \Gamma(f)$ $\cup \Gamma(e f) \cup \Gamma(f e) \cup \Gamma(f e f) \cup \Gamma(e f e)$, and that $S(e, f) \cap E=\{e, f, g=E \cap \Gamma(e f)$, $h=E \cap \Gamma(f e), f g=h f, e h=g e\}$. Note further that $(e f)^{n} \in e S \cap S f$ for each $n$, so that $g \in e S \cap S f$, and $e g=g=g f$. Similarly $f h=h=h e$. Let $K$ be the kernel of $S(e, f)$. By (3), $K$ is a group with unit $u$ and it is easy to see that $u p=u=p u$ for each idempotent $p$ of $S(e, f)$. It is then clear that $u$ is a zero for $S(e, f)$ since it annihilates the generators $e$ and $f$.

(4) implies (1). Let $e, f \in E$; we use the notation and facts introduced in the first part of $(3) \rightarrow(4)$, and consider six cases.

Case 1. $e=u$, the zero of $S(e, f)$. Then $(e f)^{n} \rightarrow e$ and $(f e)^{n} \rightarrow e$.

Case 2. $f=u$; then $(e f)^{n} \rightarrow f$ and $(f e)^{n} \rightarrow f$.

Case 3. $g=u$; then since $g$ is a zero of $S(e, f)$ and $g \in \Gamma(e f)$, we have $(e f)^{n} \rightarrow g$. Hence $(f e)^{n}=f(e f)^{n-1} e \rightarrow f g e=g$.

Case 4. $h=u$. This is similar to Case 3.

Case 5. $f g=h f=u$. Then $g=g g=(g f) g=g(f g)=g u=u$, and Case 3 applies.

Case 6. $e h=g e=u$. This is similar to Case 5, and the proof is complete.

Definition. (a) If $e, f \in E$ we say that $e \geqq f$ if $e f=f e=e$, i.e., if $e \in f S f$.

(b) + is a quasi-involution on $S$ if $+: E \cdot E \rightarrow S$ satisfies $e, f \in E \rightarrow(e f)^{+} \in f S e$ $=f S \cap S e$, and $e^{+}=e$ for all $e \in E$. If such a function exists, $S$ has $q . i$.

THEOREM 3. The following are equivalent for a compact semigroup $S$ :

(1) $S$ has q.i.

(2) If $e$ and $f$ are idempotents, and $g$ is the idempotent in $\Gamma(e f)$ then $g \geqq e$ and $g \geqq f$.

(3) If $e$ and $f$ are idempotents then there exists an idempotent $g$ such that $(e f)^{n} \rightarrow g$ and $(f e)^{n} \rightarrow g$.

Proof. (1) implies (2). Let + be a quasi-involution on $S, e, f \in E$, and $g \in E \cap$ $\Gamma(e f)$. Then, as noted in the proof of (3) implies (4) of Theorem 2,eg=g and $g f=g$. By (1), $g=g^{+}=(e g)^{+} \in g S e$ and $g=g^{+}=(g f)^{+} \in f S g$. It follows that $g=g e=f g$, and hence that $e g=g=g e$ and $g f=g=f g$. This establishes (2). 
(2) implies (3). Let $e, f \in E$; by (2) we have $g^{2}=g \in \Gamma(e f)$ satisfying $g \geqq e$ and $g \geqq f$. Since $g$ annihilates both $e$ and $f$ it follows that $g$ is a zero for $\Gamma(e f)$, and hence that $(e f)^{n} \rightarrow g$. Then $(f e)^{n}=f(e f)^{n-1} e \rightarrow f g e=g$, so (3) holds.

(3) implies (1). Define, for $e, f \in E,(e f)^{+}=\lim (e f)^{n}=\lim (f e)^{n}$. To see that + is well defined, let $e_{1}, f_{1}, e_{2}, f_{2} \in E$ be such that $e_{1} f_{1}=e_{2} f_{2}$. Then for some $g \in E$, $\lim \left(f_{1} e_{1}\right)^{n}=g=\lim \left(e_{1} f_{1}\right)^{n}=\lim \left(e_{2} f_{2}\right)^{n}$. It follows from (3) that $g=\lim \left(f_{2} e_{2}\right)^{n}$, and hence that $\left(e_{1} f_{1}\right)^{+}=\left(e_{2} f_{2}\right)^{+}$, so + is well defined. Now for $e, f \in E$, and $\lim (e f)^{n}=g=\lim (f e)^{n}$ it is clear that $g \in f S \cap S e$. Finally for $e \in E, e^{+}=(e e)^{+}$ $=\lim (e e)^{n}=e$, and the proof is complete.

The remainder of this section is devoted to establishing relations between $S$ and $\tilde{S}$.

LEMMA 1. Let $G \subset S$ be a compact semigroup. Let $G^{\prime}$ be the set of all $v \in \tilde{S}$ with $C(v) \subset G$. Then $G^{\prime}$ (endowed with the relative weak-* topology from $\tilde{S}$ ) is isomorphic with $\tilde{G}$. It follows that if $G$ is a group with Haar measure $\mu$, then $\mu$ is a zero for $G^{\prime}$.

Proof. The equivalence of $G^{\prime}$ and $\tilde{G}$ is well known and easy to verify. The fact that $\mu$ is a zero for $G^{\prime}$ follows from Wendel [11, p. 925].

THEOREM 4. $S$ has q.i. if and only if $\tilde{S}$ has q.i.

Proof. Suppose $\tilde{S}$ has q.i., and let $e, f \in E$. Then $e, f \in E(\tilde{S})$ so by Theorem 3 and the fact that $S$ is a closed subsemigroup of $\tilde{S}$, there exists $g \in E$ with $(e f)^{n}$ $\rightarrow g$ and $(f e)^{n} \rightarrow g$. Thus $S$ has q.i. by Theorem 3. Now suppose $S$ has q.i. and let $\mu, v \in E(\widetilde{S})$, and $\{\mathscr{T}\}=E(\tilde{S}) \cap \Gamma(\mu v)$. We will show that $\mathscr{T} \geqq \mu$, v. Let $A=C(\mu)$, $B=C(v)$, and $C=C(\mathscr{T})$. Since $\mu \mathscr{T}=\mathscr{T}=\mathscr{T} v$ we have [6, Lemma 2.1] $A C=C$ $=C B$. Note also that $\mathscr{T} \mu \in E(\tilde{S})$ with $C(\mathscr{T} \mu)=C A$ and $v \mathscr{T} \in E(\tilde{S})$ with $C(v \mathscr{T})=B C$. It then follows from previous results that $C A, A, B, C$, and $B C$ are groups with Haar measures $\mathscr{T} \mu, \mu, v, \mathscr{T}$ and $\nu \mathscr{T}$ respectively. Now let $e, f$, and $g$ respectively be the units of $A, C$, and $B$. Then ef $\in A C=C$, and since $S$ has q.i. it follows that $(e f)^{n} \rightarrow f$ and that $f \geqq e$. Similarly $f \geqq g$, so $e f=f=f e$ and $g f=f=f g$. For $c \in C, c=c f=c(f e)=(c f) e=c e$ and $c=f c=(g f) c=g(f c)=g c$ so $e$ is a right unit for $C$ and $g$ is a left unit for $C$. Since $C(f e)=f e \in C A$ and $C(g f)=g f \in B C$ it follows from Lemma 1 that $\mathscr{T} \mu f e=\mathscr{T} \mu$ and $g f v \mathscr{T}=\nu \mathscr{T}$. By a similar argument (since $C(\mu f) \subset A C=C$ and $C(f v) \subset C B=C$ ) it follows that $\mathscr{T} \mu f=\mathscr{T}$ and $f v \mathscr{T}=\mathscr{T}$. Thus $\mathscr{T} \mu=\mathscr{T} \mu f e=(\mathscr{T} \mu f) e=\mathscr{T} e$ and $v \mathscr{T}=g f v \mathscr{T}=g(f v \mathscr{T})=g \mathscr{T}$. Now if $F$ is a continuous complex function on $S$ we have

$$
\begin{aligned}
(\mathscr{T} e)(F) & =\int F(x e) d \mathscr{T}(x)=\int_{C} F(x e) d \mathscr{T}(x)=\int_{C} F(x) d \mathscr{T}(x) \\
& =\int F(x) d \mathscr{T}(x)=\mathscr{T}(F)
\end{aligned}
$$

i.e., $\mathscr{T} \mu=\mathscr{T} e=\mathscr{T}$. Similarly $v \mathscr{T}=g \mathscr{T}=\mathscr{T}$. Thus $\mathscr{T} \geqq \mu$ and $\mathscr{T} \geqq v$ and by Theorem 3, $\tilde{S}$ has q.i. The proof is complete. 
We now consider some relations between the $\mathscr{D}$-class structures of $S$ and of $\tilde{S}$.

LEMMA 2. Let $\phi \in \tilde{S}$ be a regular element. Then $C(\phi)$ is contained in a single regular $\mathscr{D}$-class $D$ of $S$. If further $\mathscr{T}$ is any inverse of $\phi$, then carrier $\mathscr{T} \subset D$, and each element a of $C(\phi)$ has an inverse $a^{\prime}$ in carrier $\mathscr{T}$. For any such $a^{\prime}$, $C(\phi) a^{\prime}$ is a minimal left ideal of the compact simple semigroup $C(\phi \mathscr{T})$, and $a^{\prime} C(\phi)$ is a minimal right ideal of the compact simple semigroup $C(\mathscr{T} \phi)$.

Proof. Let $\mathscr{T}$ be an inverse of $\phi$, so that $\phi=\phi \mathscr{T} \phi$ and $\mathscr{T}=\mathscr{T} \phi \mathscr{T}$. We show first that each element of $C(\phi)$ has an inverse in $C(\mathscr{T})$. If $\alpha=\phi \mathscr{T}$, then $\alpha^{2}=\alpha$ so $C(\alpha)$ is a compact simple semigroup of $S$. Let $a \in C(\phi)=C(\phi \mathscr{T} \phi)$ $=C(\alpha) C(\phi)=C(\phi) C(\mathscr{T}) C(\phi)$. Then $a=p d q$ with $p \in C(\phi), d \in C(\mathscr{T})$, and $q \in C(\phi)$. Since $p d \in C(\alpha)$, a compact simple semigroup, there exist unique idempotents $e$ and $f$ in $C(\alpha)$ with $p d \in e C(\alpha) e$ and $q d \in f C(\alpha) f$ and the latter are maximal groups of $C(\alpha)$. Since $a=p d q, a d=p d q d \in p d C(\alpha)$ and hence $a d C(\alpha) \subset p d C(\alpha)^{2}=p d C(\alpha)$. Since these are minimal right ideals of $C(\alpha)$ we conclude that $\operatorname{ad} C(\alpha)=p d C(\alpha)$. Then $e C(\alpha)=p d C(\alpha)=a d C(\alpha)$ and similarly $C(\alpha) f=C(\alpha) q d=C(\alpha) a d$, so that $a d \in C(\alpha) \cdot f \cap e C(\alpha)$, a maximal group of $C(\alpha)$ with unit $g$. Since $C(\alpha) f=C(\alpha) \cdot g$ and $e C(\alpha)=g C(\alpha)$ and $a=p d q$, it follows that $g a=g$. Let $(a d)^{\prime}$ denote the inverse of ad in the group $g C(\alpha) g$. Then $a\left[d(a d)^{\prime}\right] a=a d(a d)^{\prime} a=g a=a$, and $d(a d)^{\prime} a d(a d)^{\prime}=d\left[(a d)^{\prime} a d\right](a d)^{\prime}$ $=d\left[g(a d)^{\prime}\right]=d(a d)^{\prime}$; i.e., $d(a d)^{\prime}$ is an inverse of $a$, and $d(a d)^{\prime} \in C(\mathscr{T}) C(\alpha)$ $=C(\mathscr{T} \phi \mathscr{T})=C(\mathscr{T})$.

Thus if $a \in C(\phi)$, there exists an inverse $a^{\prime}$ of $a$ in $C(\mathscr{T})$. By Corollary 1 of [9], each of $a, a^{\prime}$ and $a a^{\prime}$ lie in $D_{a}$ and also the compact simple semigroup $C(\phi \mathscr{T}) \subset D_{a}$. By a similar argument $b \in C(\phi)$ implies $C(\phi \mathscr{T}) \subset D_{b}$. Since $D_{a}$ meets $D_{b}$ we have $D_{a}=D_{b}$, so $C(\phi) \subset D\left(=D_{a}=D_{b}\right)$. Since $a^{\prime} \in C(\mathscr{T})$ has an inverse $a \in C(\phi)$ it is clear also that $C(\mathscr{T}) \subset D$.

To complete the lemma, let $a \in C(\phi)$ and let $a^{\prime}$ be an inverse of $a$ in $C(\mathscr{T})$. Let $\alpha=\phi \mathscr{T}$ and $\beta=\mathscr{T} \phi$; both $\alpha, \beta \in E(\tilde{S})$ so $C(\alpha)$ and $C(\beta)$ are compact simple semigroups. Further, $C(\phi) a^{\prime} \subset C(\phi) C(\mathscr{T})=C(\alpha)$, and if $x=p q$ with $p \in C(\phi)$, $q \in C(\mathscr{T})$ then $x C(\phi) a^{\prime} \subset C(\phi \mathscr{T} \phi) a^{\prime}=C(\phi) a^{\prime}$. Hence $C(\phi) a^{\prime}$ is a left ideal of $C(\alpha)$. But $C(\phi) a^{\prime}=C(\phi) a^{\prime} a a^{\prime} \subset C(\alpha) a a^{\prime}$ and $a a^{\prime} \in C(\alpha)$ imply that $C(\phi) a^{\prime}$ $=C(\alpha) a a^{\prime}$ by minimality. Similarly $a^{\prime} C(\phi)$ is a minimal right ideal of $C(\beta)$, and the proof is complete.

If $S$ (or equivalently $\tilde{S}$ ) has q.i., we denote by $a^{\prime}$ and $\phi^{\prime}$ respectively the (unique by Theorems $2,3,4$ ) inverses of the regular elements $a \in S$ and $\phi \in \tilde{S}$. Also if $A \subset S$ and $B \subset \tilde{S}$ each consist of regular elements, $A^{\prime}$ and $B^{\prime}$ respectively denote the set of inverses of the elements of $A$ and $B$. We define carrier $B$, where $B \subset \tilde{S}$ to be the closure in $S$ of $\bigcup C(\phi): \phi \in B]$ and use the notation $C(B)$.

LEMMA 3. If $\phi$ is a regular element of $\tilde{S}$, and $S$ has q.i. then $C(\phi)^{\prime}=C\left(\phi^{\prime}\right)$. 
Proof. By Lemma 2, $C(\phi)^{\prime} \subset C\left(\phi^{\prime}\right)$ ! and $C\left(\phi^{\prime}\right)^{\prime} \subset C(\phi)$. Thus $C(\phi)$ $=\left[C(\phi)^{\prime}\right]^{\prime} \subset C\left(\phi^{\prime}\right)^{\prime} \subset C(\phi)$, so $C(\phi)=C\left(\phi^{\prime}\right)^{\prime}$ and $C(\phi)^{\prime}=C\left(\phi^{\prime}\right)$.

THEOREM 5. The following are equivalent:

(1) $S$ has q.i.

(2) If $\Gamma$ is a regular $\mathscr{H}$-class in $\tilde{S}$ then $C(\Gamma)$ is contained in a single $\mathscr{H}$-class of $S$.

(3) If $\phi$ and $\psi$ are regular with $C(\phi)=C(\psi)$, then $\phi \mathscr{H} \psi$.

Proof. (1) implies (2). Fix $\phi \in \Gamma$ and let $\alpha=\phi \phi^{\prime}, \beta=\phi^{\prime} \phi$. Then by (1) and Theorems 3 and 2, each of $C(\alpha)$ and $C(\beta)$ is a group. Let $e$ be the unit of $C(\alpha)$ and let $f$ be the unit of $C(\beta)$. By Lemma 3, $C(\alpha)=C(\phi) C(\phi)^{\prime}$ and $C(\beta)=C(\phi)^{\prime}$ - $C(\phi)$. Now let $x \in C(\phi)$; then $x x^{\prime} \in E \cap C(\alpha)$ and $x^{\prime} x \in E \cap C(\beta)$, so $x \in C(\phi)$ implies $x x^{\prime}=e$ and $x^{\prime} x=f$. Thus $x \mathscr{R} e$ and $x \mathscr{L} f$, i.e., $x \in R_{e} \cap L_{f}=H$ and $H$ is an $\mathscr{H}$-class of $S$. Hence $C(\phi) \subset H$ and since $H$ is closed it follows that $C(\Gamma) \subset H$.

(2) implies (1). Let $\mu^{2}=\mu \in \tilde{S}$; we will show that $C(\mu)$ is a group. Since $\mu$ is regular, $\Gamma=H_{\mu}$ is a regular $\mathscr{H}$-class $[9$, p. 275], so by (2), there exists an $\mathscr{H}$-class $H$ of $S$ with $C(\Gamma) \subset H$. Hence $H \cap E \neq \square$ and it follows [9, p. 275] that $H$ is a group. Thus $C(\mu)$ has a single idempotent and is therefore a group.

(1) implies (3). Let $\phi$ and $\psi$ be regular elements of $S$ with the same carrier and let $\mu=\phi \phi^{\prime}$ and $v=\phi^{\prime} \phi$. Then $C(\mu)=C(\phi) C(\phi)^{\prime}=C(\psi) C(\psi)^{\prime}=C(\psi) C\left(\psi^{\prime}\right)$ $=C\left(\psi \psi^{\prime}\right)=C(v)$. Thus $\mu$ and $v$ are idempotents with the same carrier and hence by Theorem $1, \mu=v$, i.e., $\phi \phi^{\prime}=\psi \psi^{\prime}$. Similarly $\phi^{\prime} \phi=\psi^{\prime} \psi$. But then $\phi \mathscr{R} \phi \phi^{\prime}, \phi \phi^{\prime} \mathscr{R} \psi \psi^{\prime}$ and $\psi \psi^{\prime} \mathscr{R} \psi$ imply $\phi \mathscr{R} \psi$. Similarly $\phi \mathscr{L} \psi$, so $\phi \mathscr{H} \psi$.

(3) implies (1). Let $\mu$ and $v$ be two idempotents with the same carrier. We will show that $\mu=v$. By (3), $\mu \mathscr{H} v$; but $\mathscr{H}$-equivalent idempotents must be equal [9, p. 275], so $\mu=v$.

The final result of this section gives, for q.i. semigroups, a structure theorem for regular $\mathscr{H}$-classes of $\tilde{S}$.

THEOREM 6. These are equivalent.

(1) $S$ has q.i.

(2) If $\Gamma$ is a regular $\mathscr{H}$-class in $\tilde{S}$ and $\alpha, \beta \in E(\tilde{S})$ with $\alpha \mathscr{R} \phi$ and $\phi \mathscr{L} \beta$ for all $\phi \in \Gamma$, then there exist compact groups $G, H \subset S$ such that $\alpha$ is Haar measure on a compact normal subgroup of $G, \beta$ is Haar measure on a compact normal subgroup of $H$, and $\Gamma=G \phi=\phi H$ for each $\phi \in \Gamma$.

Proof. (1) implies (2). Let $\Gamma, \alpha$, and $\beta$ be as in (2). By [9, Theorem 3], we have that $\Gamma=H_{\phi}=H_{\alpha \phi}=H_{\alpha} \cdot \phi$ and $\Gamma=H_{\phi}=H_{\phi \beta}=\phi H_{\beta}$. Since $H_{\alpha}$ and $H_{\beta}$ are compact groups in $\tilde{S}$, the Glicksberg structure theorem gives compact groups $G$ and $H$ contained in $S$ and idempotents $\mu$ and $v$ with $\mu=$ Haar measure on a compact subgroup of $G, v=$ Haar measure on a compact subgroup of $H$, and $H_{\alpha}=\mu G=G \mu$ and $H_{\beta}=\nu H=H v$. It is easy to verify, using the invariance of 
$\mu$ and $v$ that $\alpha=\mu$ and $\beta=v$; i.e., $\Gamma=(G \alpha) \phi=G(\alpha \phi)=G \phi$ and $\Gamma=\phi(\beta H)$ $=(\phi \beta) H=\phi H$ for each $\phi \in \Gamma$. Thus (2) holds.

(2) implies (1). Let $\mu \in E(\tilde{S})$; then $\Gamma=H_{\mu}$ is a regular $\mathscr{H}$-class of $\tilde{S}$ with $\phi \mathscr{R} \mu$ and $\phi \mathscr{L} \mu$ for each $\phi \in \Gamma$. Take $\alpha=\mu=\beta$ in (2) and we see that $\mu$ is Haar measure on some group; in particular, $C(\mu)$ is a group. By Theorem 1, the proof is complete.

4. Involutions on semigroups. We again restrict our attention to compact topological semigroups $S$. If $T$ is a subsemigroup of $S$, a mapping $+: T \rightarrow T$ is called an involution on $T$ provided $a, b \in T$ implies $\left(a^{+}\right)^{+}=a$ and $(a b)^{+}=b^{+} a^{+}$. Certain additional restrictions which may be placed on + are:

(A) + is continuous.

(B) $e^{2}=e$ implies $e^{+}=e$.

(C) $G$ a compact group, $G \subset T$ implies $G^{+} \subset G$.

(D) $G$ a compact group, $G \subset T$ implies $+\mid G$ coincides with inversion in $G$.

We observe that any involution + satisfying $B$, and defined on a semigroup containing $E$, defines a quasi-involution. Indeed, if one requires only that $(a b)^{+}=b^{+} a^{+}$and $e^{+}=e$ for all $a, b \in T$ and $e \in E$, then $e, f \in E$ implies $(e f)^{+}=f^{+} e^{+}=f e \in f S e$, and $e^{+}=e$ so + is a quasi-involution. Thus, involutions satisfying (B) defined on a semigroup containing $E$ are of particular interest. One other type of involution is of primary interest here, namely one which is defined on all regular elements of $S$.

We first prove several lemmas needed in the sequel. If $T \subset S$ is a semigroup, then $T$ is an inverse semigroup if each element of $T$ has a unique inverse in $T$. It is known (e.g., see [9, p. 273]) that $T$ is an inverse semigroup if and only if each element of $T$ is regular, and the idempotents of $T$ commute.

LEMMA 4. Let $T$ be a compact semigroup of $S$ containing all regular elements of $S$, and let + be an involution on $T$. Then $e^{+}=e$ for each $e \in E$ if and only if $H_{e}^{+} \subset H_{e}$ for each $e \in E$, where $H_{e}$ is the maximal subgroup of $S$ containing $e$. In either case, if $a$ is regular then $a^{\prime}$ and $a^{+}$lie in the same $\mathscr{H}$-class of $S$, and $a$ regular $\mathscr{H}$-class $H$ contains a self involutory element $\left(b=b^{+}\right)$if and only if $H$ is a group.

Proof. Let $a$ be a regular element, and $D$ the $\mathscr{D}$-class containg $a$. Assume first that + satisfies $B$. By [9, Lemma 3], there exist $e, f \in E \cap D$ such that $f \in R_{a}$ and $e \in L_{a}$, hence $S e=S a$ and $f S=a S$. In particular $a=a e$ and $e=x a$ for some $x \in S$. But $(x f) a=x(f a)=x a=e$ and $x f S=x(f S)=x(a S)=x a S=e S$; in particular, $x f \mathscr{R} e, x f \in D$, and we may assume that $x a=e$ with $x \in D \subset T$. Hence $a^{+}=(a e)^{+}=e^{+} a^{+}=e a^{+}$and similarly $e=e^{+}=(x a)^{+}=a^{+} x^{+}$. From these equalities we obtain $a^{+} S=e a^{+} S \subset e S=a^{+} x^{+} S \subset a^{+} S$. Thus $a^{+} S=e S$ and $a^{+} \mathscr{R} e$. In a similar way we see that $a^{+} \mathscr{L} f$, so $a^{+} \in R_{e} \cap L_{f}$. Now $S$ has a quasi-involution, so $a$ has a unique inverse $a^{\prime} \in R_{e} \cap L_{f}$ and hence $a^{+} \mathscr{H} a^{\prime}$. Now let $x \in H_{e}$, where $e^{2}=e$; then $x^{+}$and $x^{\prime}$ belong to $H_{e}$, since $H_{e}$ contains an in- 
verse of $x$. Hence $x \in H_{e}$ implies $x^{+} \in H_{e}$, so $H_{e}^{+} \subset H_{e}$. Conversely if $H_{e}^{+} \subset H_{e}$, then $e e=e$ implies $e^{+} e^{+}=e^{+} \in H_{e}$, and the idempotent $e^{+}$must then be $e$.

Finally, if an $\mathscr{H}$-class $H$ is a group, then the idempotent of $H$ is self involutory. Conversely if a regular $\mathscr{H}$-class $H$ contains an element $x$ with $x^{+}=x$, then $x$ and $x^{\prime}$ are in $H$, so $H$ is a group [9, Corollary 4].

THEOREM 7. Let $T$ be the: closed semigroup of $S$ generated by the maximal groups of $S$. The following are equivalent: (1) $S$ has q.i. and the product of any two idempotents is regular, (2) $E$ is commutative, (3) $T$ is an inverse semigroup.

Proof. (1) implies (2). We show that $E^{2} \subset E$. It will then follow that if $e, f \in E$, then $f e$ is an inverse of $e f$ and hence $f e=e f$. Fix $e, f \in E$. By (1), ef is regular so there exists $a \in S$ such that efaef $=$ ef and aefa $=a$. Then (multiplying the last equation on the left by $f), f a(e f) f a=f a e f a=f a$. Also, ef $(f a) e f=e f a e f=e f$, i.e., $f a$ is an inverse of $e f$. By the uniqueness of inverses in $S, f a=a$. Similarly $a=a e$, so $f a=a=a e$. But then $a^{2}=(a e)(f a)=a e f a=a$, i.e., $a \in E$. Hence $a=a^{\prime}=e f$; i.e., $e f \in E$.

(2) implies (3). It suffices to show that each element of $T$ has an inverse in $T$. Let $e, f \in E, p \in H_{e}$ and $q \in H_{f}$, and denote by $p^{-1}$ and $q^{-1}$ the respective inverses of $p$ and $q$ in $H_{e}$ and $H_{f}$. Then $p q\left(q^{-1} p^{-1}\right) p q=p f e q=p e f q=p q$ and $q^{-1} p^{-1}$ $(p q) q^{-1} p^{-1}=q^{-1} e f p^{-1}=q^{-1} f e p^{-1}=q^{-1} p^{-1}$, i.e., $q^{-1} p^{-1}$ is an inverse of $p q$, with $q^{-1} p^{-1} \in T$. It follows that each element of the semigroup generated by the maximal groups of $S$ has an inverse in $T$. Using the compactness of $S$, this property easily extends to the closure.

(3) implies (1). From the theorem quoted prior to Lemma 4, the idempotents of $T$ commute. Since $E \subset T, E$ is commutative, and the proof is complete (since (2) easily implies (1)).

We say that the convergence theorem holds in $\tilde{S}$ if the following are equivalent for $v \in \tilde{S}:$ (1) $\left\{v^{n}\right\}$ converges, (2) $\lim \sup C\left(v^{n}\right)=\liminf C\left(v^{n}\right),(3) C(v) \subset C(\mu)$ where $\mu^{2}=\mu \in K(v)$. It was shown in [5, Theorem 1] that the convergence theorem holds in $\tilde{S}$ when $S$ is a group.

THEOREM 8. The following are equivalent: (1) $S$ is a group, (2) $S$ is an inverse semigroup and there exists a regular element of $\tilde{S}$ whose carrier is $S$, (3) $S$ is an inverse semigroup, the convergence theorem holds in $\tilde{S}$, and there exists $\phi \in \tilde{S}$ with $C(\phi)=S$, (4) $S$ has q.i., the convergence theorem holds in $\tilde{S}$ and there is a regular element of $\tilde{S}$ whose carrier is $S$.

Proof. (1) implies (2). This is clear, using Haar measure on $S$ as the regular element. To see (2) implies (1), let $\phi$ be regular with $C(\phi)=S$. Since $S$ is an inverse semigroup, both $S$ and $\tilde{S}$ have q.i. Thus $\phi$ has a unique inverse $\phi^{\prime}$, and by Lemma 3, $C\left(\phi^{\prime}\right)=C(\phi)^{\prime}$. Let $\alpha$ be the idempotent $\phi \phi^{\prime}$. Then $C(\alpha)=C(\phi) C(\phi)^{\prime}$ $=S S^{\prime}=S S=S$. Then $S$ is simple, and it follows from q.i. that $S$ is a group. 
(1) implies (3). This follows from the fact that the convergence theorem holds in $\tilde{S}$ when $S$ is a group.

(3) implies (4). It suffices to show that there exists a regular element of $\tilde{S}$ whose carrier is $S$. Let $C(\phi)=S$. Then $\liminf C\left(\phi^{n}\right)=\lim \sup C\left(\phi^{n}\right)=S$. Now $\left\{\phi^{n}\right\}$ converges to $\mu=\mu^{2}$ and $S=C(\phi) \subset C(\mu)$; i.e., $C(\mu)=S$.

(4) implies (1). By Lemma 2, if $\phi$ is regular with $C(\phi)=S$, then each element of $S$ is regular, so by q.i., $S$ is an inverse semigroup. Now, as in (3) implies (4), $S=C(\phi) \subset C(\mu)$ where $\mu^{2}=\mu$. Hence $S$ is simple, $S$ is a group, and the proof is complete.

THEOREM 9. Let + be a continuous involution on a compact semigroup $T$ containing all regular elements of $S$. Let $\tilde{T}$ denote the set of all $\phi \in \tilde{S}$ with $C(\phi) \subset T$, and define for $\mu \in \tilde{T}, \mu^{*}(f)=\int_{T} f\left(x^{+}\right) d \mu(x)$ where $f$ is continuous and complex valued. Then $*$ is a continuous involution on $\tilde{T}, \phi \in \tilde{T}$ implies $C\left(\phi^{*}\right)$ $=C(\phi)^{+}$, and $\tilde{T}$ contains all regular elements of $\tilde{S}$. Finally, the following conditions are equivalent: (1) * satisfies (B), (2) + satisfies (C), and (3) * satisfies (C).

Proof. It is clear that $\phi \in \tilde{T}$ implies $\phi^{*} \in \tilde{S}$, and we show first that $C\left(\phi^{*}\right)$ $=C(\phi)^{+}$. Let $f$ be continuous with $f \mid C(\phi)^{+}=0$. Then $f\left(x^{+}\right)=0$ for each $x \in C(\phi)$, so $\phi^{*}(f)=\int_{T} f\left(x^{+}\right) d \phi(x)=\int_{C(\phi)} f\left(x^{+}\right) d \phi(x)=0$. It follows that $C\left(\phi^{*}\right) \subset C(\phi)^{+}$. But then $\phi^{* *}(f)=\int_{T} f\left(x^{+}\right) d \phi^{*}(x)=\int_{T} f\left(x^{++}\right) d \phi(x)$ $=\int_{T} f(x) d \phi(x)=\int_{s} f(x) d \phi(x)=\phi(f)$, so $\phi^{* *}=\phi$.

By the first part of the proof $C(\phi)=C\left(\phi^{* *}\right) \subset C\left(\phi^{*}\right)^{+}$, so $C(\phi)^{+} \subset C\left(\phi^{*}\right)$ and they are equal. The fact that $\tilde{T}$ contains all regular elements of $\tilde{S}$ follows from Lemma 2.

If now $\mu, v \in \tilde{T}$, then $\mu v \in \tilde{T}$ by Lemma 1 , and $(\mu v)^{*}(f)=\int_{T} f\left(x^{+}\right)(d \mu v)(x)$. Let $\bar{f}$ be a fixed continuous extension to $S$ of the function $f^{+}: T \rightarrow$ complexes defined by $f^{+}(t)=f\left(t^{+}\right)$. Then

$$
\begin{gathered}
\int_{T} f\left(x^{+}\right) d\left(\mu v(x)=\int_{S} \tilde{f}(x) d(\mu v)(x)=\iint \tilde{f}(x y) d \mu(x) d v(y)\right. \\
=\int_{T} \int_{T} \tilde{f}(x y) d \mu(x) d v(y)=\int_{T} \int_{T} f\left[(x y)^{+}\right] d \mu(x) d v(y)=\int_{T} \int_{T} f\left(y^{+} x^{+}\right) d \mu(x) d v(y) .
\end{gathered}
$$

But the latter is, by Fubini's theorem, $\int_{T} \int_{T} f\left(y^{+} x^{+}\right) d v(y) d \mu(x)$, and this is $\left(v^{*} \mu^{*}\right)(f)$; i.e., $(\mu v)^{*}=v^{*} \mu^{*}$, so ${ }^{*}$ is an involution on $\tilde{T}$. To show ${ }^{*}$ is continuous on $\tilde{T}$, let $\left\{\mu_{\alpha}\right\}$ be a net in $\tilde{T}$, and let $\mu \in \tilde{T}$ with $\mu_{\alpha} \rightarrow \mu$. Then $\mu_{\alpha}^{*}(f)$ $=\int_{T} f\left(x^{+}\right) d \mu_{\alpha}(x)=\int_{S} \bar{f}(x) d \mu_{\alpha}(x) \rightarrow \int_{S} \bar{f}(x) d \mu(x)=\int_{T} f\left(x^{+}\right) d \mu(x)=\mu^{*}(f)$, so $*$ is continuous.

We show next that $*$ satisfies (B) if and only if + satisfies (C). Suppose + satisfies (C), and let $\phi^{2}=\phi \in \tilde{S}$. Then $\phi \in \tilde{T}$, and $\left(\phi^{*}\right)^{2}(f)=\iint f(x y) d \phi^{*}(x) d \phi^{*}(y)$ $=\int_{T} \int_{T} f\left(x^{+} y^{+}\right) d \phi(x) d \phi(y)=\int_{T} \int_{T} f\left[(y x)^{+}\right] d \phi(x) d \phi(y)=\int_{T} \int_{T} f^{+}(y x) d \phi(x) d \phi(y)$ $=\int_{T} \int_{T} f^{+}(y x) d \phi(y) d \phi(x)=\phi^{2}\left(f^{+}\right)=\phi\left(f^{+}\right)=\phi^{*}(f)$; i.e., $\phi^{*} \in E(\tilde{S})$. Note further, 
if $G=C\left(\phi^{*}\right)$ (a compact group), that $C\left(\phi^{*}\right)=C(\phi)^{*}=G^{+}=G=C(\phi)$. By the uniqueness of Haar measure $\phi=\phi^{*}$, and * satisfies (B). Conversely, suppose * satisfies (B), and fix a compact group $G \subset S$. Let $\phi$ be Haar measure on $G$. Then $\phi^{*}=\phi$, so $G=C(\phi)=C\left(\phi^{*}\right)=C(\phi)^{+}=G^{+}$, and + satisfies (C).

To complete the proof, suppose ${ }^{*}$ satisfies (B), and let $\Gamma$ be a compact subgroup of $\widetilde{S}$. Since ${ }^{*}$ is a quasi-involution on $\tilde{S}$, there exists a compact group $G \subset S$ with $\Gamma=\mu G=G \mu$, where $\mu^{2}=\mu \in \Gamma$. But then, if $\phi \in \Gamma$, there exists $x \in G$ with $\phi=\mu x$, so $\phi^{*}=(\mu x)^{*}=x^{*} \mu^{*}=x^{*} \mu$. Now $x^{*}(f)=\int f\left(t^{+}\right) d x(t)$ $=f\left(x^{+}\right)=x^{+}(f)$, so $x^{*}=x^{+}$. Hence $x^{+} \in G$ (since $*$ satisfies (B) implies + satisfies (C)). Thus $\phi^{*}=x^{*} \mu=x^{+} \mu \in G \mu=\Gamma$, and $\Gamma^{*} \subset \Gamma$. The proof is complete.

THEOREM 10. Let + be a continuous involution on a compact semigroup $T$ containing the regular elements of $S$, and let $*$ be the involution on $\tilde{T}$ defined in Theorem 9. Then + satisfies (D) if and only if * satisfies (D); in either case both $*$ and + satisfy (B) and (C) as well. Further, if either + or $*$ satisfies (D) and $a$ and $\phi$ are regular elements (of $S$ and $\tilde{S}$, respectively) then $a a^{+}, a^{+} a$, $\phi \phi^{*}$, and $\phi^{*} \phi$ are self-involutory elements of order two, and $\phi^{\prime}=\phi^{*} \phi \phi^{*}, a^{\prime}$ $=a^{+} a a^{+},\left(a^{+}\right)^{\prime}=\left(a^{\prime}\right)^{+}$, and $\left(\phi^{*}\right)^{\prime}=\left(\phi^{\prime}\right)^{*}$.

Proof. It is clear that (D) implies (C), and hence (B), for any involution. Now suppose + satisfies (D) and let $\Gamma$ be a compact subgroup of $\tilde{S}$. As before, $\Gamma=\mu G=G \mu$, where $\mu^{2}=\mu \in \Gamma$. If $\phi=\mu x \in \Gamma$, then $\phi^{*}=x^{+} \mu=x^{-1} \mu$, where $x^{-1}$ denotes the inverse of $x$ in the group $G$. But then $\phi \phi^{*}=\mu x x^{-1} \mu=\mu e \mu=\mu^{2}$ $=\mu$, since $\mu e=\mu$ ( $\mu$ being right invariant on a subgroup of $G$ containing the unit $e$ of $G$ ). Similarly, $\phi^{*} \phi=x^{-1} \mu \mu x=x^{-1} \mu x=\mu x^{-1} x=\mu e=\mu$, since $\mu$ commutes with each element of $G$. Thus $\phi^{*}$ is the inverse of $\phi$ in $\Gamma$, and * satisfies (D). Conversely, suppose * satisfies (D), and let $G$ be a compact group in $S$. Then $G$ is also a compact group in $\tilde{S}$, so $x \in G$ implies $x^{+}=x^{*}=x^{-1}$; i.e., + satisfies (D).

Now suppose either + or ${ }^{*}$ satisfies (D), and let $a$ be a regular element in $S$. It is clear that $a a^{+}$and $a^{+} a$ are self-involutory elements of $S$. Now by Lemma 4, $a^{+}$and $a^{\prime}$ are in the same $\mathscr{H}$-class of $S$, and it follows from Theorem 3 of [9] that $a a^{+}$and $e=a a^{\prime}$ are in the same $\mathscr{H}$-class; similarly for $a^{+} a$ and $f=a^{\prime} a$. However, $H_{e}$ and $H_{f}$ are groups [9, Corollary 2], and $a a^{+}$and $a^{+} a$ are self involutory elements thereof. It follows that $a a^{+}=\left(a a^{+}\right)^{+}=\left(a a^{+}\right)^{-1}$ (inverse in $H_{e}$ ) and $a^{+} a=\left(a^{+} a\right)^{+}=\left(a^{+} a\right)^{-1}$ (inverse in $\left.H_{f}\right)$. Hence $\left(a a^{+}\right)^{2}=e$ and $\left(a^{+} a\right)^{2}=f$. From this we have $a\left(a^{+} a a^{+}\right) a=a$, and $\left(a^{+} a a^{+}\right) a\left(a^{+} a a^{+}\right)$ $=a^{+}\left(a a^{+} a a^{+} a\right) a^{+}=a^{+} a a^{+}$, i.e., $a^{\prime}=a^{+} a a^{+}$. But then $\left(a^{\prime}\right)^{+}=\left(a^{+} a a^{+}\right)^{+}$ $=a a^{+} a$; we complete the proof by showing that $a a^{+} a$ is the inverse of $a^{+}$. Note that $a^{+}\left(a a^{+} a\right) a^{+}=a^{+}\left(a a^{+} a a^{+}\right)=a^{+} e=a^{+}$, and $\left(a a^{+} a\right) a^{+}\left(a a^{+} a\right)=a\left(a^{+} a a^{+} a\right)$ $a^{+} a=a f a^{+} a=(a f) a^{+} a=a a^{+} a$, and the proof is complete.

We note that by a similar argument it can be shown that any regular self involutory element is of order two. 
It may be of interest to correlate the involution discussed in the previous theorem with some previous work along these lines by Wendel [11, p. 924]. In this particular situation $S$ was a compact group and the involution ${ }^{*}$ defined by Wendel was $\mu^{*}(f)=\int f\left(x^{-1}\right) d \mu(x)$; i.e., this * is the * defined in Theorem 9 , taking $x^{+}=x^{-1}$. Wendel showed that his ${ }^{*}$ was an involution on $\tilde{S}$ satisfying (A) and (B). From Theorem 10 it follows that * satisfies (D) as well. A generalization of Wendel's involution is obtained by requiring $S$ to be an inverse semigroup. In this case we note that ${ }^{*}$ is an affine mapping on $\tilde{S}$; i.e., $\left(\sum_{i-1}^{n} a_{i} \phi_{i}\right)^{*}$ $=\sum_{i=1}^{n} a_{i} \phi_{i}^{*}$ for all $n \geqq 1, \phi_{i} \in \tilde{S}$, and $a_{i} \geqq 0$ with $\sum_{i=1}^{n} a_{i}=1$.

THEOREM 11. Let $S$ be an inverse semigroup and let ${ }^{*}$ be the involution on $\tilde{S}$ defined in Theorem 9 (taking $x^{+}=x^{\prime}$ ). Then * is the unique continuous affine involution on $\tilde{S}$ which satisfies (D) and coincides with' on $S$.

Proof. It is obvious that ${ }^{*}$ satisfies these conditions, and the restrictions imposed insure that any other such involution must necessarily agree with * on the set of convex combinations of point measures. This set is dense in $\tilde{S}[1$, Lemmas 3.1 and 3.2], and thus by continuity the involutions agree on $\tilde{S}$.

\section{BIBLIOGRAPHY}

1. R. F. Arens and J. L. Kelley, Characterizations of the space of continuous functions over a compact Hausdorff space, Trans. Amer. Math. Soc. 62 (1947), 499-508.

2. A. H. Clifford, Semigroups containing minimal ideals, Amer. J. Math. 70 (1948), 521-526.

3. H. S. Collins, Remarks on affine semigroups, Pacific J. Math. (to appear). 442-446.

4. - Idempotent measures on compact semigroups, Proc. Amer. Math. Soc. 13 (1962),

5. - Convergence of convolution iterates of measures, (submitted).

6. Irving Glicksberg, Convolution semigroups of measures, Pacific J. Math. 9 (1959), 51-67.

7. J. A. Green, On the structure of semigroups, Ann. of Math. (2) 54 (1951), 163-172.

8. R. J. Koch, On monothetic semigroups, Proc. Amer. Math. Soc. 8 (1957), 397-401.

9. D. D. Miller and A. H. Clifford, Regular $\mathscr{D}$-classes in semigroups, Trans. Amer. Math. Soc. 82 (1956), 270-280.

10. A. D. Wallace, The structure of topological semigroups, Bull. Amer. Math. Soc. 61 (1955), 95-112.

11. J. G. Wendel, Haar measure and the semigroup of measures on a compact group, Proc. Amer. Math. Soc. 5 (1954), 923-929.

Louisiana State University, Baton Rouge, Louisiana 